\section{Carga de doença da COVID-19 e de suas complicações agudas e crônicas: reflexões sobre a mensuração (DALY) e perspectivas no Sistema Único de Saúde}

\author{
Burden of disease from COVID-19 and its \\ acute and chronic complications: reflections on \\ measurement (DALYs) and prospects for the \\ Brazilian Unified National Health System
}

\section{Carga de enfermedad de la COVID-19 y de sus complicaciones agudas y crónicas: reflexiones sobre la medición (DALY) y perspectivas en el Sistema Único de Salud de Brasil}

\author{
Mônica Rodrigues Campos 1 \\ Joyce Mendes de Andrade Schramm 1 \\ Isabel Cristina Martins Emmerick 2 \\ Jéssica Muzy Rodrigues 1 \\ Fernando Genovez de Avelar 3 \\ Thiago Goes Pimentel 1
}

doi: 10.1590/0102-311X00148920

\title{
Resumo
}

A COVID-19 é uma síndrome respiratória aguda grave (SRAG) infecciosa, causada por coronavírus. A doença tem alta transmissibilidade e ocasiona sintomas leves a graves, gerando elevada demanda por cuidados intensivos e milhares de óbitos. Em março de 2020, a COVID-19 foi caracterizada como pandemia e já soma mais de 5 milhões de casos e 300 mil óbitos pelo mundo. A história natural da doença ainda não é bem estabelecida, dificultando a elaboração de protocolos clínicos eficazes e medidas de prevenção. Apesar disso, pode-se afirmar que é uma doença de abordagem sistêmica, já que há evidências de complicações agudas e crônicas, além de efeitos catastróficos na saúde mental da população. Destaca-se então a necessidade de uma metodologia que capte de forma mais efetiva os efeitos da COVID-19, considerando aspectos como sua gravidade, duração e potencial de gerar complicações crônicas que aumentarão as demandas no Sistema Único de Saúde (SUS). Nesse sentido, é de extrema utilidade o indicador DALY, ou anos de vida perdidos por morte prematura ajustados por incapacidade (DALY), que agrega a (1) mortalidade - estimativa dos anos de vida perdidos (YLL) e (2) morbidade - estimativa dos anos vividos com incapacidade (YLD). Este artigo discute a relevância e as dificuldades de estudar a carga da COVID-19 e de suas complicações, no contexto brasileiro, ressaltando a importância de caracterizar a história natural da doença e estimar indicadores como o YLD, que considerem a alta carga de morbidade no planejamento de estratégias para lidar com as consequências da COVID-19 pós-pandemia. Discute-se também os desafios futuros para o enfrentamento da doença no SUS e reflexões sobre o cálculo do DALY.

Infecções por Coronavírus; Anos Potenciais de Vida Perdidos; Indicadores de Morbimortalidade; Atenção Primária à Saúde

\author{
Correspondência \\ J. M. Rodrigues \\ Rua José Higino 11, Rio de Janeiro, RJ 20520-201, Brasil. \\ jehmuzy@gmail.com \\ 1 Escola Nacional de Saúde Pública Sergio Arouca, Fundação \\ Oswaldo Cruz, Rio de Janeiro, Brasil. \\ 2 University of Massachusetts Medical School, Worcester, U.S.A. \\ ${ }^{3}$ Escola Nacional de Saúde Pública, Universidade NOVA de \\ Lisboa, Lisboa, Portugal.
}




\section{Definição e relevância da COVID-19}

Em dezembro de 2019 foi registrado no escritório da Organização Mundial da Saúde (OMS), na China 1, o primeiro caso oficial de pneumonia, ainda com causa desconhecida, em Wuhan, província de Hubei, despertando particular interesse das autoridades sanitárias. Ao final de janeiro de 2020, foi decretado estado de emergência em saúde pública por se tratar de uma calamidade com interesse internacional. Em fevereiro de 2020, a síndrome respiratória aguda grave (SRAG) que crescia em progressão exponencial foi nomeada pela OMS como COVID-19 1, sendo em 11 de março de 2020 caracterizada pela mesma como pandemia. Até esse momento, a COVID-19 estava presente em cerca de 100 países, com mais de 100 mil casos confirmados da doença, sendo necessárias medidas específicas de identificação, prevenção e controle para o seu enfrentamento 1,2,3.

A COVID-19 é uma SRAG infecciosa causada por coronavírus, sendo representada pelo agente etiológico SARS-CoV-2. Outras epidemias já foram relatadas por agentes etiológicos semelhantes, como o SARS-CoV-1 e o MERS, entretanto nenhuma delas com tamanha magnitude 2. O SARS-CoV-2 apresentou uma particular rapidez e disseminação de novos casos.

Em Wuhan, o indicador de transmissibilidade $\left(\mathrm{R}_{0}\right)$, que aponta a velocidade de disseminação do vírus em humanos foi de 2,2 (IC95\%: 1,4; 3,9), indicando que cada pessoa infectada pode infectar outras duas pessoas, em média, em um contato efetivo 4 . Na recente literatura, o $\mathrm{R}_{0}$ do SARS-CoV-2 variou de 1,95 (IC95\%: 1,4; 2,5) 1 a 6,5 (IC95\%: 5,7; 7,2) 4, sendo o $\mathrm{R}_{0}$ médio estimado de 3,3, com mediana de 2,8 4. A variação observada deve-se a estimativas em momentos diferentes de deflagração da doença 1,4 .

No que concerne à sua patogênese, de $70 \%$ a $80 \%$ dos infectados serão assintomáticos ou apresentarão sintomas leves da doença. Ainda sem consenso na literatura, é estimado que $20 \%$ desenvolverão a forma mais grave da doença em cuidados hospitalares e podendo chegar a $5 \%-10 \%$ de cuidados intensivos 1,5. O período médio de convalescência é de 19 dias (de 2 a 5 dias até o aparecimento dos sintomas e 14 dias pós-sintomas até a recuperação), porém os casos sob cuidados intensivos levam de 3 a 6 semanas ${ }^{1}$. Como toda a população é suscetível, um aumento muito rápido do número de infectados ocasionará sobrecarga de leitos, procedimentos e equipamentos hospitalares 5,6. Quanto aos seus fatores de riscos associados à gravidade, destaca-se a idade avançada (maior que 60 anos, principalmente para o risco aumentado de mortalidade) e a presença de comorbidades (doenças cardiovasculares, obesidade, diabetes, hipertensão, doenças pulmonares etc.), acelerando o progresso dos sintomas com pior prognóstico 4.

Segundo dados da OMS (https://www.covidvisualizer.com, acessado em 30/Mai/2020), foram identificados em nível mundial aproximadamente 6 milhões de casos confirmados da doença, com mais de 365 mil mortes associadas a esta patologia. Na mesma data, o Ministério da Saúde brasileiro apresentou oficialmente cerca de 470 mil casos confirmados (incidência de 221,5 por 100 mil habitantes) e quase 30 mil mortes por essa doença, com uma taxa de letalidade de 6\% (taxa de mortalidade de 13,3 por 100 mil habitantes), tendo o registro do primeiro caso ocorrido em 26 de fevereiro de 2020 e a primeira morte oficial em 17 de março de 2020 (Ministério da Saúde. https://covid.saude.gov.br/, acessado em 30/Mai/2020). Ressalta-se que o presente cenário deve-se à adesão nacional ao protocolo de distanciamento social, recomendado pela OMS e adotado desde 12 de março de 20207.

\section{Mensuração do DALY da COVID-19: reflexões, limites e possibilidades}

A despeito da importância que a mensuração e análise das taxas de morbimortalidade possam ter para a compreensão do impacto da COVID-19, registra-se a relevância de uma metodologia que compute de forma mais efetiva o quanto este agravo influencia as condições de saúde da população, considerando aspectos como sua gravidade, duração, potencial de gerar complicações crônicas por faixa etária, sexo e local de ocorrência, além do impacto no Sistema Único de Saúde (SUS) da evolução da doença.

Nesse sentido, é de extrema utilidade o indicador "anos de vida perdidos por morte prematura ajustados por incapacidade” (disability-adjusted life year - DALY), desenvolvido por Murray \& Lopez 8,9 em meados da década de 1990, no contexto do estudo Carga Global de Doença (Global Burden of Disease 
- GBD). Esse indicador é dividido em dois componentes: (1) mortalidade - estimativa dos anos de vida perdidos por morte prematura (years of life lost - YLL) e (2) morbidade - estimativa dos anos vividos com incapacidade (years lived with disability - YLD).

Passadas quase três décadas de seu surgimento, os estudos de carga global de doença 9 têm incorporado atualizações e refinamentos metodológicos 10,11. Ao longo desse período, intensos debates ocorreram sobre a proposta metodológica da métrica DALY, vários refinamentos metodológicos foram incorporados. Além disso, agregou pesquisadores, grupos de pesquisa e instituições de todo o mundo como colaboradores das várias edições das estimativas nacionais/mundiais do GBD, sob a coordenação de Christopher Murray, que dirige o core group do Instituto de Métricas e Avaliação de Saúde, Universidade de Washington (Seattle, Estados Unidos). Houve um aumento significativo do número de doenças e sequelas avaliadas, bem como dos fatores de risco investigados 8,10,12,13.

Os desafios impostos e o surgimento/ressurgimento de novas e antigas doenças impõem atualizações, novas metodologias para estimar a carga de morbidade (YLD), muitas vezes com grau importante de incompletude tendo em vista o limitado conhecimento sobre a história natural da doença, a exemplo das arboviroses, mais especificamente a Zika e chikungunya e, no momento, a pandemia da COVID-19. Além disso, as questões relacionadas às especificidades, tendo em conta as características econômicas, demográficas e desigualdades sociais, continuam a ser debate para a reflexão sobre as estimativas nacionais/globais.

No Brasil, foram realizadas duas edições do estudo carga de doença para o país: o primeiro realizado no ano de 1998 14,15,16 e o segundo em 2008 17,18,19; e estudos para morbidades específicas como: diabetes 20,21 , hepatites/cirrose 22,23,24, causas externas $25,26,27$ e doenças negligenciadas 28 . A última versão do estudo nacional traz refinamentos metodológicos que pontuam a originalidade dos estudos nacionais, representando uma importante diferença com relação ao GBD, mais expressivamente em relação ao componente de morbidade (YLD). Nesse sentido, destaca-se que as duas versões do estudo de Carga de Doença Brasil (1998 14 e 2008 17) não podem ser comparadas diretamente, sendo necessária a replicação dos aportes metodológicos para cada morbidade 26 .

No caso da COVID-19, o panorama clínico-epidemiológico acerca das complicações agudas e crônicas requer relativo tempo para execução. A literatura científica disponível e necessária para a realização das estimativas do YLD, tendo em vista exíguo período pós-COVID-19 para a investigação dos casos recuperados, aponta desafios a serem enfrentados no cálculo do DALY: o mapeamento das complicações agudas e crônicas da COVID-19 e os seus respectivos parâmetros clínico-epidemiológicos.

O DALY é sensível à qualidade/precisão das informações utilizadas como fonte de dados para a obtenção dos inputs à estimativa, bem como do conjunto de pesos atribuídos à gravidade das complicações crônicas mensuradas (medidas a serem construídas), o que introduz atualmente um grau de incerteza relevante para o DALY da COVID-19. Logo, são relevantes os estudos de análise de sensibilidade que avaliam a incerteza observada nas estimativas, considerando os diferentes cenários de inputs e processo decisório, por exemplo, em relação à atribuição de pesos e/ou fontes de informação 29; bem como à ponderação pela presença e quantidade de comorbidades associadas, que tamanha variabilidade traz ao YLL 30. Nesse sentido, dois cenários de possíveis fontes poderiam ser considerados: (a) dados oficias das Secratarias Estaduais de Saúde/Ministério da Saúde (Ministério da Saúde. https://covid.saude.gov.br/, acessado em 30/Mai/2020) e (b) fontes alternativas: SIVEP-Gripe (http://info.gripe.fiocruz.br, acessado em 30/Mai/2020) e Registro de Óbitos (Portal da Transparência. Painel registral. https://transparencia.registrocivil.org.br/ especial-covid, acessado em 23/Abr/2020). Assim, com base nessas composições de cenários poderiam ser então realizados conjuntos de análise de sensibilidade: dados oficiais vs corrigidos por subnotificação, que implicariam certamente diferenças no conjunto final do DALY.

O indicador DALY tem ainda como vantagem a análise específica por estrutura etária, fator de risco significativo para COVID-19, agregada à presença/quantidade de comorbidades, como visto no estudo de Hanlon et al. 30, que demonstra importante variação de YLL por idade, segundo a presença de outros diagnóstico pré-existentes crônicos. Por exemplo, tem-se 35 YLLs (médios) para homens de 50-59 anos (sem comorbidades), enquanto que 19 YLLs, para cinco comorbidades, em razão da menor esperança de vida e menor incidência deste grupo com mais comorbidades. Por outro lado, para aqueles com 80 e mais a perda é de 11 YLLs (sem comorbidades) e de 1,5 YLLs para 11 comorbidades, a despeito do maior risco e maior incidência deste grupo etário. 
Outro ponto relevante no processo decisório para o cálculo do YLD é o desafio da definição do conjunto de pesos atribuídos à gravidade das complicações agudas e crônicas associadas à COVID-19, dado o atual desconhecimento da completa HND, seu horizonte clínico-epidemiológico (que afetará a duração das mesmas), mudanças no protocolo terapêutico de tratamento devido a inovações tecnológicas advindas de estudos emergentes de âmbito mundial, tanto para aferir eficácia de medicamentos conhecidos quanto para a busca de novos fármacos e vacinas (que implicará o indicador de porcentagem de tratados, também incorporado ao YLD).

Todas essas questões terão impacto direto na mensuração do YLD, que traduz o tempo vivido com uma incapacidade associada à morbidade de base aferida, dado o acesso ao protocolo de tratamento, ponderado pela gravidade (peso) de cada complicação aguda/crônica 8,9 .

\section{Problemas atuais e futuros: complicações agudas e crônicas da COVID-19 e a construção da métrica para a carga da COVID-19}

Os elementos essenciais para o cálculo do YLD dizem respeito à incorporação do tempo "perdido" decorrente das complicações e respectivas gravidades que é contabilizado por meio dos pesos das incapacidades e duração de complicações agudas e crônicas. Nesse sentido, ainda que não se esgotem as possíveis complicações decorrentes da COVID-19, em função da necessidade de mais estudos no que tange ao tropismo celular e mecanismos patogênicos 31,32, esta trata-se de uma doença de abordagem sistêmica, com evidências de possíveis complicações em órgãos vitais. Assim, a disseminação da COVID-19 no corpo é extensa e por consequência os seus efeitos 33,34,35.

$\mathrm{O}$ agente etiológico SARS-CoV-2 ao entrar no organismo hospedeiro se liga ao receptor da enzima conversora de angiotensina 2 (ECA2), permitindo a entrada na célula-alvo e replicação desencadeando uma resposta imunológica no hospedeiro, surgindo os primeiros sintomas e manifestações clínicas 35. A despeito de ser reconhecido o tropismo desse no trato respiratório superior e no tecido pulmonar, em decorrência da porta de entrada, outros órgãos que também expressam esse receptor podem ser acometidos 36 e assim o indivíduo poderá desenvolver outras manifestações clínicas correspondentes 37.

Para entender a distribuição da doença no corpo humano, Wu et al. 38 realizaram estudos sobre alterações metabolômicas e lipidômicas em que demonstraram aparente correlação destas com o desenvolvimento da COVID-19, indicando que a mesma alterou o metabolismo disperso em todo o corpo, com reflexos desde o nível celular aos diversos sistemas orgânicos.

Essas alterações metabólicas, em decorrência da relativa susceptibilidade subjacente ao processo de infecção por desregulação endotelial, devido ao mecanismo inflamatório causado, reduzem a capacidade dos vasos de desempenhar funções regulatórias importantes 31,37. Assim, podem acarretar complicações agudas ou crônicas que estão relacionadas às formas mais graves da doença 30,31,37,39,40.

$\mathrm{Na}$ literatura científica atual, os órgãos-alvo já relatados incluem pulmões, mas a falta de oxigênio e a inflamação generalizada também podem danificar de forma aguda os rins (27\%) 41, fígado (50\%) 42 , trato gastrointestinal (20\%) 43,44,45, causar alterações na cascata de coagulação e sistema hematopoiético, coração e sistema cardiovascular 46, cérebro e sistema nervoso central (SNC) 47 e outros órgãos 41.

Quanto às complicações agudas 36, a propensão do desenvolvimento de coágulos sanguíneos a partir de inflamações nestes mesmos vasos, especialmente nos pulmões, pode ser responsável pelo desenvolvimento de formas mais graves da doença, uma vez que estes trombos podem desempenhar um papel direto e significativo nas anormalidades das trocas gasosas e na disfunção de órgãos multissistêmicos 31,32,37.

No cérebro e SNC, as sequelas neurológicas podem ser devastadoras, especialmente em decorrência de infecções virais respiratórias, uma vez que já é conhecida pelo menos duas rotas de entrada no SNC, via hematogênica mediada por receptores ECA2 e por vias retrógadas neuronais, e consequente infecção induzida por vírus neuropático, o que pode explicar o aumento da ocorrência de acidente vascular cerebral (AVC), mudanças de comportamento e anosmia 31.

No que concerne ao coração e sistema vascular, as complicações mais relatadas são lesão cardíaca aguda, insuficiência cardíaca, miocardite, inflamação vascular e arritmias cardíacas 39,40,48. Os problemas cardíacos identificados, associados a um aumento dos valores encontrados nas enzimas cardíacas, 
podem estar relacionados à combinação de uma resposta inflamatória sistêmica significativa e a uma inflamação vascular localizada no nível da placa arterial 46.

Quanto ao fígado, foi identificada insuficiência hepática, com enzimas hepáticas de valores até 3 vezes superiores ao de referência 35,48, mais comum em homens 33,49 e em casos mais graves 33,48. Foram identificadas esteatose microvascular moderada e atividade lobular e portal leve, entretanto ressalta-se que para além da infecção mediada por SARS-CoV-2, o comprometimento hepático pode estar relacionado com uma indução promovida pela polimedicação 33,35,49. Até o momento não há evidências robustas que suportem insuficiência hepática crônica nesses pacientes 33 , a despeito de alguns estudos 39,49 .

A insuficiência renal nos pacientes acometidos com COVID-19 ocorreu pelo aumento da creatinina sérica e redução da taxa de filtração glomerular. A infecção por SARS-CoV-2 pode induzir a necrose tubular aguda grave e infiltração de linfócitos, causando mais danos tubulares por meio do recrutamento de macrófagos para se infiltrar no túbulo interstício $34,48,50$, podendo ser explicado pela expressão dos receptores ECA2 nos túbulos renais 34,50.

Foram identificadas alterações trombóticas em pacientes com COVID-19 32,37,51,52, a trombocitopenia foi associada a cinco vezes mais chances de desenvolver a forma mais grave da doença 49,50. Estudos apontam que a COVID-19 pode aumentar o risco de desenvolver coagulação intravascular disseminada 51. Pode-se inferir que a desregulação da cascata de coagulação e a consequente formação de coágulos intra-alveolares ou sistêmico de fibrina são proeminentes em achados de pacientes não apenas com COVID-19, mas também com outras doenças respiratórias graves 51 .

Vê-se, então, que o vírus atua em um receptor que está envolvido no controle do sistema circulatório, agindo nas pequenas artérias, o que leva a inúmeras complicações agudas 36 . Logo, no tocante ao cálculo do YLD, é necessário considerar que as complicações apontadas demandam quase que um "estudo de carga de doença completo", com levantamento de parâmetros clínico- epidemiológicos relativos a cada uma dessas condições. Uma estimativa "mais simplificada" seria agregar os casos por gravidades diferenciadas e assim estimar o YLD de COVID-19 para: casos leves, moderados, graves/ UTI, graves com complicações. O que de toda forma traz uma importante complexidade à estimativa. $\mathrm{O}$ mesmo, obviamente, se estende às complicações crônicas, no tocante à estimativa da duração e pesos de incapacidades.

Nesse cenário em que a história natural da doença está em constante evolução, é ainda impossível determinar todas as complicações crônicas que os sobreviventes da COVID-19 enfrentarão. Assim, não se pode descartar que tais complicações estarão relacionadas àquelas agudas relatadas anteriormente, tais como insuficiência renal, AVC, insuficiência hepática, entre outras 37,38,39,47,48,51.

Visando a mapear quais seriam as complicações crônicas advindas da COVID-19 e compreender o seu desencadeamento, pode-se considerar os estudos das pneumonias graves que evoluem para a síndrome de angústia respiratória aguda (SARA) 53, levando a cicatrizes que em geral causam problemas respiratórios a longo prazo e que aumentam o risco de ataque cardíaco e derrame 47 . Verificou-se que pacientes pós-internação por pneumonia grave com SARA apresentaram cerca de 4 vezes mais riscos de ataque cardíaco e derrame no primeiro ano e 1,5 nos 9 anos subsequentes.

Os pacientes gravíssimos da COVID-19, que passam muito tempo em UTI, ficam propensos a desenvolver a "síndrome de cuidados intensivos" 53,54,55,56, que caracteriza-se por um conjunto de alterações físicas (atrofia e fraqueza muscular - 50\%), cognitivas (79\%) e mentais (28\%) que reduzem a qualidade de vida tanto do paciente quanto de seus cuidadores 53,55 .

Quanto ao comprometimento cognitivo, os fatores de risco associados a este processo contemplam a duração do delírio na UTI, disfunção cerebral aguda (AVC, alcoolismo), hipóxia (SARA, parada cardíaca), hipotensão (sepse grave, trauma), insuficiência respiratória que requer ventilação mecânica prolongada e uso de terapia de substituição renal 54,55 .

Em relação às alterações físicas, a fraqueza neuromuscular adquirida na UTI é a forma mais comum deste comprometimento, ocorrendo em mais de $25 \%$ dos sobreviventes da UTI, com mobilidade reduzida, quedas recorrentes ou tetraparesia 53,54 .

No que se refere às alterações mentais, o risco de desenvolver alterações psicológicas pode passar de $60 \% 54$ e inclui sintomas como ansiedade, depressão e estresse pós-traumático 53,54,55.

Face ao exposto, melhorar a qualidade de vida para o paciente com alta hospitalar pós-UTI engloba uma série de medidas, tais como: fisioterapia pós-admissão, cuidados nutricionais e apoio 
psicológico 54. Isso reforça a tese do impacto sobre os cuidadores e aumento dos custos associados ao cuidado na convalescência do indivíduo acometido pela COVID-19.

Conforme relatado antes, o SARS-CoV-2 devido à potencial formação de coágulos sistêmicos 31,37 pode reduzir o fluxo sanguíneo para o cérebro, provocando complicações neurológicas do SNC, como convulsões, perda de consciência, perda de olfato (5\% a 10\%) e "intenso e prolongado delírio", que pode acarretar prejuízos cognitivos a longo prazo, como déficits de memória 47,53,54,56. Na COVID-19, tal delírio pode ser agravado pelo uso de sedativos (benzodiazepínicos) 57 para o enfrentamento das crises violentas de tosse e a angústia e desconforto do tubo respiratório 47,53,54,56. Na emergente literatura, ressaltam-se ainda as seguintes complicações: sintomas similares à síndrome de Kawasaki em crianças 58,59, casos reportados que apresentaram síndrome de Guillain-Barré 60,61,62,63, além de complicações de retina 64 e nos testículos, com possíveis efeitos sobre a fertilidade 65.

Dessa forma, evidencia-se a importância de que sejam desenvolvidos estudos para a mensuração da carga de doença da COVID-19, posto que comorbidades temporárias e permanentes representam uma demanda crescente dos serviços de saúde para o acompanhamento desses pacientes, seja no nível da atenção primária ou média complexidade, que historicamente apresenta limites ao acesso dos pacientes, com impacto no diagnóstico, monitoramento e reabilitação.

Até o presente momento, grau de desconhecimento sobre os determinantes e complicações da mesma é um elemento que compromete o processo decisório da construção dos parâmetros clínico-epidemiológicos para o cálculo do YLD, bem como da definição dos pesos de gravidade associados, que tanto influenciarão na sensibilidade da medida.

\section{Carga de saúde mental por COVID-19: para onde vamos?}

Além das complicações agudas e crônicas mencionadas no tópico anterior, a pandemia da COVID-19 cria um cenário mundial de fatores de risco também para problemas de saúde mental no curto e longo prazos. Esse quadro impacta tanto pacientes (devido ao isolamento social, quarentena e/ou tratamento médico da COVID-19, em especial, hospitalização prolongada e cuidados intensivos) quanto a população sob distanciamento social 7, criando um ambiente propício a problemas de saúde, tais como: ansiedade, depressão, sentimento de desamparo e incertezas sobre o futuro 66,67.

Cabe então um olhar diferenciado no que tange à saúde mental de grupos de interesse com riscos diferenciados, a saber: profissionais de saúde, pacientes com diagnóstico psiquiátrico prévio, pacientes recuperados da COVID-19 ou população em geral sem diagnóstico de saúde mental, passíveis de tornarem-se casos clínicos 68 .

Profissionais de setores essenciais, em especial os da saúde, serão sobrecarregados por um ambiente de trabalho estressante, contribuindo com o desenvolvimento de transtornos mentais, tais como: ansiedade, síndrome de estresse pós-traumático (SEPT) e burnout 66,67,69. Esse cenário que comporta um aumento expressivo de demanda por atendimento psiquiátrico, bem como por assistência aos diagnosticados com COVID-19, leva os profissionais de saúde a demandarem cuidados de saúde mental para si, na medida em que se desgastam em sua atividade laboral. Esse quadro pode vir a acarretar o seu afastamento e perda de produtividade, reduzindo assim a capacidade de atendimento do sistema de saúde, conforme adoecem. O cuidado com a saúde dos profissionais dos setores essenciais na pandemia é fundamental para minimizar seu desgaste e, consequentemente, garantir melhores condições para o exercício de suas atividades.

No que concerne aos pacientes acometidos de transtornos mentais, que encontravam-se em tratamento psiquiátrico antes do surgimento da COVID-19, estes têm o seu acesso aos serviços de saúde mental dificultado pela pandemia, em função do distanciamento social e da sobrecarga do sistema de saúde, além de, possivelmente, ter seu quadro clínico agravado pelo sentimento de solidão e isolamento $66,67,69$. Em especial nos pacientes graves, sequelas duradouras ou permanentes podem ocorrer, o que, por sua vez, pode acarretar transtornos mentais relativos à perda de capacidade física 70 .

Na perspectiva da população, em especial nos países em desenvolvimento como o Brasil, os impactos econômicos das medidas preventivas da transmissão da doença estão sendo sentidos e continuarão refletidos no aumento da pobreza, do desemprego e da população sem moradia 69 . Assim, sob tal situação, condições preexistentes de saúde mental, ainda não tratadas, podem ser agravadas, ou novas 
surgirem, o que por sua vez nos leva a inferir no aumento de depressão, ansiedade, abuso de substâncias psicoativas, automutilação e tentativas de suicídio 69,71. Ou seja, a piora das condições socioeconômicas pode levar ao aumento da prevalência de complicações mentais durante e pós-pandemia.

Adicionalmente, o cenário atual de estratégia de controle da COVID-19 pode acirrar conflitos familiares e isolar o convívio da família dos ambientes externos, favorecendo o aumento de casos de violências domésticas e abusos de vulneráveis 66,69,70,71, o que ocasiona ambientes inóspitos que podem ser propícios ao desenvolvimento de traumas psicológicos.

Como estratégia deve-se pensar em uma reestruturação na dinâmica do cuidado à saúde mental em tempos de pandemia, em que atendimentos psicológicos on-line e abordagens de atendimento coletivo passam a ser considerados possíveis alternativas para lidar com o aumento desta demanda 72,73.

A pandemia atinge, então, toda a sociedade, gerando um espaço favorável ao surgimento de transtornos mentais 66,67,68,69,70,71,72 e, por isto, não é absurdo cogitar uma pandemia de transtornos mentais após/durante a pandemia de COVID-19.

Nesse sentido, ao considerar que a carga de saúde mental historicamente agrega a maior parcela de YLD (cerca de 50\%) 8,10,14,18 e que na atual conjuntura pandêmica de COVID-19 há um cenário de intensificação deste grupo de morbidades crônicas, faz-se então necessária a investigação dos parâmetros clinico-epidemiológicos para a mensuração destas condições (YLD).

Cabe ressaltar que o indicador DALY, conforme exposto anteriormente, é composto por duas parcelas, mortalidade (YLL) e morbidade (YLD). Entretanto, na programação/gestão do sistema de saúde tradicionalmente utiliza-se apenas o indicador mortalidade, o que não é efetivo no que tange à saúde mental. Dessa forma, evidencia-se a necessidade da mensuração do YLD que impactará na programação de recursos humanos e capacidade instalada para o atendimento por estas morbidades, uma vez que a construção deste indicador é capaz de evocar o peso deste componente, tão expressivo nestes grupos.

\section{Desafios futuros para o enfrentamento da COVID-19 no SUS e reflexões sobre o DALY}

É bem conhecida na literatura as estimativas da carga global de doença sobre a importância do indicador DALY em medir em uma mesma métrica a mortalidade e a morbidade. Pode-se mesmo apontar que o componente que mede as incapacidades temporárias e permanentes representa o eixo estratégico para subsidiar o planejamento, a programação e o impacto econômico no sistema de saúde, sendo possível expor as diferenças entre o SUS e o sistema privado.

Os estudos de carga de doença realizados no país para os anos de referência 199814 e 200817 e as estimativas realizadas pela OMS para os anos 2000, 2010 e 201612 apresentaram resultados para o componente de morbidade (YLD), como um todo, de cerca de 50\% 12,13,75. Esse percentual significa que a fração correspondente à metade da carga de doença no Brasil expressa o peso de se conviver com as sequelas temporárias e permanentes geradas pelas doenças incluídas nos estudos. O que observase é que expressiva parte do conjunto de sequelas são complicações advindas das doenças crônicas não transmissíveis o que, no entanto, não exclui as complicações relacionadas às doenças infecciosas crônicas como a doença de Chagas, a aids, a Zika e, em particular, aquelas relacionadas à COVID-19 ainda não completamente definidas, tendo em vista o parcial conhecimento da história natural da COVID-19.

As complicações oriundas das doenças variam com o grau de limitação e comprometimento da autonomia das pessoas, influenciam na qualidade de vida gerando muitas vezes aposentadorias precoces e aumento da demanda pela atenção de média e alta complexidades que, por sua vez, tem distribuição desigual no país limitando ainda mais o acesso aos serviços, diagnóstico tardio e capacidade de reabilitação limitada, apesar do SUS garantir a universalidade e integralidade do acesso ao sistema de saúde 76. A aprovação da Emenda Constitucional no 95 (EC95) foi um elemento na redução do investimento em saúde para um SUS subfinanciado ao longo dos anos 77, trazendo a redução da qualidade dos serviços públicos de saúde e limitando ainda mais o acesso dos pacientes. Esse cenário foi agravado pela redução do investimento em políticas de proteção e promoção social, bem como trazendo o fechamento de clínicas da família e a volta de doenças previamente eliminadas, tal como o sarampo 78 . 
A epidemia de COVID-19 atinge o país em um cenário de desemprego, empobrecimento e outros fatores de risco que estão relacionados à transmissibilidade da doença. As crises econômicas são elementos que podem ser facilitadores para a transmissão de doenças infecciosas e dificultar a implementação das ações de controle que em contextos de pandemia podem expressar uma mortalidade comparável a situações de guerra, piorando a crise econômica 78. Para Sands et al. ${ }^{79}$, as doenças infecciosas representam um dos maiores riscos para o crescimento econômico, embora o impacto da recessão econômica no controle das doenças infecciosas dependa do contexto, do mapeamento das áreas de risco, da situação de vulnerabilidade e decisão política de financiamento, como é o caso de uma possível revogação do teto de gastos, visando a um aumento dos recursos necessários para reforçar o orçamento da saúde e da proteção social 80 .

As diferentes condições individuais e coletivas (socioeconômicas e demográficas) para o enfrentamento da crise de saúde pública provocada pela COVID-19 são bastante desiguais no país. Dados apresentados por um estudo nacional 81 revelam que enquanto as regiões Sul e Sudeste concentram, proporcionalmente, a maioria absoluta de recursos humanos especialistas (médicos intensivistas e pneumologistas) e equipamentos hospitalares (UTIs e ventiladores mecânicos), as regiões Norte e Nordeste apresentam inferiores parâmetros em relação à sua representatividade populacional. $\mathrm{O}$ estudo sugere, assim, que dada essa discrepância de disponibilidade de capacidade instalada e de recursos humanos, a resposta ao enfrentamento da crise é desigual, impactando nas taxas de letalidade pela COVID-19 e no colapso do SUS.

Adicionalmente, em relação às diferenças sociodemográficas quanto à resposta à pandemia, o estudo amostral de soroprevalência nacional EPICOVID 82 demonstra significativa diferenciação entre raça declarada: 0,7\% para brancos, 2,3\% para pardos e 4,3\% para indígenas.

Em um contexto mais específico, no Município do Rio de Janeiro a letalidade por COVID-19 foi de $12 \%$, variando de 9,2\% nos bairros "sem favelas" a 19,5\% naqueles com "altíssima concentração de favelas" (+ de 50\% de favelas no bairro). A alta letalidade por COVID-19 nesses territórios vulneráveis pode indicar uma baixa testagem, assim como uma maior gravidade associada a doenças preexistentes ou fatores de risco, além da dificuldade de acesso à saúde 83.

As medidas de controle da pandemia terão consequências substanciais a curto e longo prazos; as restrições de distanciamento físico e quarentena reduzirão a atividade física e aumentarão outros estilos de vida não saudáveis, acarretando um aumento dos fatores de risco (tabagismo, alcoolismo, sedentarismo e obesidade) para as doenças crônicas não transmissíveis, com o agravamento dos sintomas clínicos. Alterações nas rotinas de assistência ambulatorial/hospitalar, tais como 84: cancelamento/adiamento de consultas ou de acompanhamento pré-natal e/ou adiamento de cirurgias eletivas, redução da cobertura e atraso no calendário vacinal, entre outros, terão implicações importantes para o cuidado da saúde da população como um todo, acarretando sobrecarga do sistema de saúde.

Vale destacar que mesmo após a superação da fase emergencial da pandemia, com o arrefecimento do número de casos e óbitos, surge ainda o desafio de lidar com o aumento da demanda no SUS por diagnóstico, tratamento e reabilitação dos pacientes recuperados, advindo das possíveis complicações crônicas da COVID-19, tais como: aumento da demanda por serviços de reabilitação devido às complicações respiratórias; consultas e psicoterapias tanto para os "novos" casos quanto para os casos psiquiátricos prévios agravados devido ao acesso dificultado durante a pandemia; sobrecarga dos atendimentos dos cardiologistas devido ao acúmulo das consultas adiadas no protocolo de controle para evitar a contaminação e o aumento da incidência de cardiopatias oriundas da COVID-19, com risco aumentado de derrame e ataque cardíaco; e agravamento do cenário de terapia medicamentosa e consultas médicas e cirurgias eletivas para doenças crônicas em decorrência da remarcação das consultas, sem renovação de receitas.

Assim, vê-se que os efeitos vigentes e futuros da atual pandemia no SUS são enormes e as métricas a serem construídas precisam considerar os aspectos aqui apontados. Dessa forma, o tempo e a história natural da doença são elementos essenciais para a construção das medidas (YLL e YLD) que compõem o DALY. Sem isso o grau de incerteza pode comprometer as estimativas do indicador. Vale ressaltar que as complicações associadas à COVID-19 não somente são somadas àquelas relacionadas às outras doenças, mas representam uma carga ainda maior para os serviços de saúde e para as famílias 85. Nesse sentido, cada vez mais o olhar sobre a importância da carga de morbidade (YLD) será estratégico na organização das redes de atenção, planejamento e programação das ações de saúde. 
Atualmente, considerando o debate acirrado referente às medidas de controle utilizadas até o momento, é necessário vislumbrar que ações de vigilância epidemiológica no território representam uma estratégia estruturante, juntamente com a atenção primária à saúde (APS), para o monitoramento da infecção por COVID-19 84,85,86. Além disso, deve-se considerar que o devido monitoramento da doença no país oferece informações essenciais para a estimativa dos parâmetros epidemiológicos clássicos que são necessários na construção do DALY.

Sugere-se como estratégia para o enfrentamento da pandemia/pós-pandemia e de forma a gerar parâmetros clínico-epidemiológicos mais precisos e confiáveis da doença e de suas sequelas, necessários ao DALY, garantir a devida ação da APS no rastreio e monitoramento dos infectados e dos seus contatos, aplicando estratégias de isolamento recomendadas pelas equipes de saúde da família e ações dos agentes comunitários de saúde (ACS), segundo as recomendações do guia de vigilância da Secretaria de Atenção Primária à Saúde (SAPS), Ministério da Saúde 86,87. Ou seja, que recomendações para a organização da atenção à COVID-19, no âmbito da atenção primária à saúde no SUS 88 , sejam seguidas a partir da identificação de casos suspeitos, com contatos domiciliares e, se possível, contatos comunitários nos últimos 14 dias para orientar o isolamento domiciliar por 14 dias, reduzindo contágios, evitando a geração simultânea de casos graves, de modo a desafogar o SUS.

Só com a APS forte o sistema pode ser capaz de achatar a curva de crescimento da pandemia e garantir suficiência de leitos de UTI 89 e um caminho alternativo mais seguro e eficiente para o enfrentamento da pandemia. Quanto à efetividade das estratégias de controle atuais, quanto mais tempo demorar a transmissão pessoa a pessoa, mais administrável será a situação pelo SUS.

Nesse sentido, segundo Vitória \& Campos 89, a adequação das diretrizes para a APS no enfrentamento da pandemia consiste em: (1) garantir acesso seguro à unidade básica de saúde (UBS), com adequação de estrutura física e UBS on-line (com efetiva separação da porta de entrada exclusiva para pacientes com COVID ou com o uso de tendas); (2) garantir segurança nas imunizações; (3) proteger os profissionais de saúde (orientar e prover uso de equipamentos de proteção individual); (4) garantir a longitudinalidade do cuidado; (5) fortalecer a abordagem familiar; (6) fortalecer a abordagem comunitária.

Em específico no item (4), sobre a longitudinalidade do cuidado, perpassam estratégias como as das centrais de teleatendimento, que evitam deslocamentos de pessoas sintomáticas que só precisam de orientação; e manter a equipe de saúde da família atenta à evolução do paciente, com baixa da saturação de $\mathrm{O}_{2}$ (sem sintomas) e/ou agravamento rápido de sua condição clínica. Já no item (5) - abordagem familiar -, ACS devem orientar as famílias para cuidar dos seus doentes de forma a fornecer alimentação, hidratação, medicamento para febre em quantidades adequadas e evitar que eles contaminem os demais. E, no item (6) - abordagem comunitária -, as UBS conhecem muito as famílias vulneráveis de seu território adscrito, aquelas com altíssimas taxas de densidade de habitantes por cômodos, para as quais, por exemplo, é necessário um local alternativo para quarentena em caso de contágio. Assim, as equipes de saúde da família têm subsídios que permitem melhor análise e interpretação de riscos e vulnerabilidades de indivíduos, famílias e comunidade.

Por fim, falhas no acesso ao diagnóstico têm impacto importante no perfil de morbimortalidade, que se reflete no aumento da demanda de serviços de média e alta complexidades. E, baseando-se em um acurado diagnóstico da epidemiologia da COVID-19, e em particular de suas complicações crônicas por meio da estimativa do DALY, é possível fornecer subsídios para a formulação de novas estratégias e políticas, com vistas ao enfrentamento da pandemia. No entanto, tal objetivo só será alcançado a partir do devido reconhecimento, valorização e financiamento do SUS e de seus profissionais, em especial da APS. 


\section{Colaboradores}

M. R. Campos, J. M. A. Schramm, I. C. M. Emmerick, J. M. Rodrigues, F. G. Avelar e T. G. Pimentel participaram da concepção e desenho, redação do artigo e revisão crítica do conteúdo final.

\section{Informações adicionais}

ORCID: Mônica Rodrigues Campos (0000-00027443-5977); Joyce Mendes de Andrade Schramm (0000-0003-1064-7484); Isabel Cristina Martins Emmerick (0000-0002-0383-2465); Jéssica Muzy Rodrigues (0000-0003-2526-2317); Fernando Genovez de Avelar (0000-0002-5411-3970); Thiago Goes Pimentel (0000-0002-2886-7015).

\section{Referências}

1. World Health Organization. Report of the WHO-China Joint Mission on Coronavirus Disease 2019 (COVID-19). https://www.who. int/docs/default-source/coronaviruse/whochina-joint-mission-on-covid-19-final-report. pdf (acessado em 20/Fev/2020).

2. Wang C, Horby PW, Hayden FG, Gao GF. A novel coronavirus outbreak of global health concern. Lancet 2020; 395:470-73.

3. Velavan TP, Meyer CG. The COVID-19 epidemic. Trop Med Int Health 2020; 25:278-80. doi:10.1111/tmi.13383.

4. Kolifarhood G, Aghaali M, Saadati HM, Taherpour N, Izadi N, Nazari SSH. Epidemiological and clinical aspects of COVID-19: a narrative review. Arch Acad Emerg Med 2020; 8:e41.

5. Wu Z, McGoogan JM. Characteristics of and important lessons from the coronavirus disease 2019 (COVID-19) outbreak in China: summary of a report of 72314 cases from the Chinese Center for Disease Control and Prevention. JAMA 2020; 323:1239-42.

6. Li Q, Guan X, Wu P, Wang X, Zhou L, Tong Y, et al. Early transmission dynamics in Wuhan, China, of novel coronavirus-infected pneumonia. N Engl J Med 2020; 382:1199-207.

7. Ministério da Saúde. Portaria no 356, de 11 de março de 2020. Dispõe sobre a regulamentação e operacionalização do disposto na Lei no 13.979 , de 6 de fevereiro de 2020, que estabelece as medidas para enfrentamento da emergência de saúde pública de importância internacional decorrente do coronavírus (COVID-19). Diário Oficial da União 2020; 12 mar.

8. Murray CJL, Lopez AD. The Global Burden of Disease: a comprehensive assessment of mortality and disability from diseases, injuries, and risk factors in 1990 and projected to 2020 summary. Geneva: World Health Organization; 1996

9. Murray CJL, Lopez AD. Global health statistics: a compendium of incidence, prevalence and mortality estimates for over 200 conditions. Geneva: World Health Organization; 1996.

10. Murray CL, Ezzati M, Flaxman AD, Lim S, Lozano R, Michaud C, et al. GBD 2010: a multiinvestigator collaboration for global comparative descriptive epidemiology. Lancet 2012; 380:2055-8

11. World Health Organization. The Global Burden of Disease: 2004 update. Geneva: World Health Organization; 2008.

12. Department of Information, Evidence and Research, World Health Organization. WHO methods and data sources for country-level causes of death 2000-2016. Geneva: World Heath Organization; 2018. 
13. GBD 2017 Disease and Injury Incidence and Prevalence Collaborators. Global, regional, and national incidence, prevalence, and years lived with disability for 354 diseases and injuries for 195 countries and territories, 19902017: a systematic analysis for the Global Burden of Disease Study 2017. Lancet 2018; 392:1789-858.

14. Gadelha AMJ, Leite IC, Valente JG, Schramm JMA, Campos MR. Relatório final do projeto Estimativa da Carga de Doença do Brasil, 1998. Rio de Janeiro: Escola Nacional de Saúde Pública Sergio Arouca, Fundação Oswaldo Cruz; 1998.

15. Schramm JMA, Oliveira AF, Leite IC, Valente JG, Gadelha AMJ, Portela MC, et al. Transição epidemiológica e o estudo de carga de doença no Brasil. Ciênc Saúde Colet 2004; 9:897-908.

16. Leite IC, Schramm JMA, Gadelha AMJ, Valente JG, Campos MR, Portela MC, et al. Comparação das informações sobre as prevalências de doenças crônicas obtidas pelo suplemento saúde da PNAD/98 e as estimadas pelo estudo Carga de Doença no Brasil. Ciênc Saúde Colet 2002; 7:733-41.

17. Leite IC, Valente J, Schramm JMA. Relatório final do projeto Carga de Doença do Brasil, 2008. Rio de Janeiro: Escola Nacional de Saúde Pública Sergio Arouca, Fundação Oswaldo Cruz; 2008.

18. Leite IC, Valente JG, Schramm JMA, Daumas $\mathrm{RP}$, Rodrigues RN, Costa MFS, et al. Burden of disease in Brazil and its regions, 2008. Cad Saúde Pública 2015; 31:1551-64.

19. Leite IC, Valente JG, Schramm J, Oliveira AF, Costa MFS, Campos MR. National and regional estimates of disability-adjusted lifeyears (DALYs) in Brazil, 2008: a systematic analysis. Lancet 2013; 381 Suppl:S83.

20. Costa AF, Flor LS, Campos MR, Oliveira AF, Costa MFS, Silva RS, et al. Carga do diabetes mellitus tipo 2 no Brasil. Cad Saúde Pública 2017; 33:e00197915.

21. Flor LS, Campos MR, Oliveira AF, Schramm JMA. Diabetes burden in Brazil: fraction attributable to overweight, obesity, and excess weight. Rev Saúde Pública 2015; 49:29.

22. Carvalho JR, Villela-Nogueira CA, Perez RM, Portugal FB, Flor LS, Campose MR, et al. Burden of chronic viral hepatitis and liver cirrhosis in Brazil - the Brazilian Global Burden of Disease Study. Ann Hepatol 2017; 16:893-900.

23. Portugal FB, Campos MR, Carvalho JR, Flor LS, Schramm JMA, Costa MFS. Carga de doença no Brasil: um olhar sobre o álcool e a cirrose não viral. Ciênc Saúde Colet 2015; 20:491-501.

24. Carvalho JR, Portugal FB, Flor LS, Campos MR, Schramm JMA. Método para estimação de prevalência de hepatites $\mathrm{B}$ e $\mathrm{C}$ crônicas e cirrose hepática - Brasil, 2008. Epidemiol Serv Saúde 2014; 23:691-700.
25. Campos MR, von Doellinger VR, Mendes LVP, Costa MFS, Pimentel TG, Schramm JMA. Morbidity and mortality associated with injuries: results of the Global Burden of Disease study in Brazil, 2008. Cad Saúde Pública 2015; 31:121-36.

26. Mendes LVP, Campos MR, von-Doellinger VR, Mota JC, Pimentel TG, Schramm JMA. A evolução da carga de causas externas no Brasil: uma comparação entre os anos de 1998 e 2008. Cad Saúde Pública 2015; 31:2169-84.

27. von-Doellinger V, Campos M, Mendes L, Schramm J. The 2008 Global Burden of Disease study in Brazil: a new methodological approach for estimation of injury morbidity. Rev Panam Salud Pública 2014; 36:368-75.

28. Schramm JMA, Campos MR, Emmerick ICM, Mendes LVP, Mota JC, Silva Júnior SHA. Spatial analysis of neglected diseases in Brazil, 2007-2009. Tempus (Brasília) 2016; 10:119-42.

29. Salje H, Tran Kiem C, Lefrancq N, Courtejoie N, Bosetti P, Paireau J, et al. Estimating the burden of SARS-CoV-2 in France. Science 2020; 369:208-11.

30. Hanlon P, Chadwick F, Shah A, Wood R, Minton J, McCartney G, et al. COVID-19 - exploring the implications of long-term condition type and extent of multimorbidity on years of life lost: a modelling study. Wellcome Open Research 2020; 23 abr. https://wellcomeopen research.org/articles/5-75.

31. Paniz-Mondolfi A, Bryce C, Grimes Z, Gordon RE, Reidy J, Lednicky J, et al. Central nervous system involvement by severe acute respiratory syndrome coronavirus -2 (SARS-CoV-2). J Med Virol 2020; 92:699-702.

32. Zhou F, Yu T, Du R, Fan G, Liu Y, Liu Z, et al. Clinical course and risk factors for mortality of adult inpatients with COVID-19 in Wuhan, China: a retrospective cohort study. Lancet 2020; 395:1054-62.

33. Sun J, Aghemo A, Forner A, Valenti L. COVID-19 and liver disease. Liver Int 2020; 40:1278-81.

34. Diao B, Wang C, Wang R, Feng Z, Tan Y, Wang $\mathrm{H}$, et al. Human kidney is a target for novel severe acute respiratory syndrome coronavirus-2 (SARS-CoV-2) infection. medRxiv 2020; 10 abr. https://www.medrxiv.org/content/10. 1101/2020.03.04.20031120v4.

35. Zhang C, Shi L, Wang F-S. Liver injury in COVID-19: management and challenges. Lancet Gastroenterol Hepatol 2020; 5:428-30.

36. Fellet J. Covid-19 não pode ser pensada só como doença respiratória, diz epidemiologista. BBC News Brasil 2020; 15 mai. https://www. bbc.com/portuguese/geral-52672009.

37. Oxley TJ, Mocco J, Majidi S, Kellner CP, Shoirah $\mathrm{H}$, Singh $\mathrm{P}$, et al. Large-vessel stroke as a presenting feature of Covid-19 in the young. $\mathrm{N}$ Engl J Med 2020; 382:e60. 
38. Wu D, Shu T, Yang X, Song J-X, Zhang M, Yao $\mathrm{C}$, et al. Plasma metabolomic and lipidomic alterations associated with COVID-19. medRxiv 2020; 26 abr. http://medrxiv.org/lookup/doi/ 10.1101/2020.04.05.20053819.

39. Chen T, Wu D, Chen H, Yan W, Yang D, Chen $\mathrm{G}$, et al. Clinical characteristics of $113 \mathrm{de}-$ ceased patients with coronavirus disease 2019: retrospective study. BMJ 2020; 368:m1091.

40. Madjid M, Safavi-Naeini P, Solomon SD, Vardeny O. Potential effects of coronaviruses on the cardiovascular system: a review. JAMA Cardiol 2020; 5:831-40.

41. Wadman M, Couzin-Frankel J, Kaiser J, Matacic C. How does coronavirus kill? Clinicians trace a ferocious rampage through the body, from brain to toes. Science 2020; $17 \mathrm{abr}$. https://www.sciencemag.org/news/2020/04/ how-does-coronavirus-kill-clinicians-traceferocious-rampage-through-body-brain-toes.

42. Xu L, Liu J, Lu M, Yang D, Zheng X. Liver injury during highly pathogenic human coronavirus infections. Liver Int 2020; 40:998-1004.

43. Gu J, Han B, Wang J. COVID-19: gastrointestinal manifestations and potential fecaloral transmission. Gastroenterology 2020; 158:1518-9.

44. Wu Y, Guo C, Tang L, Hong Z, Zhou J, Dong X, et al. Prolonged presence of SARS-CoV-2 viral RNA in faecal samples. Lancet Gastroenterol Hepatol 2020; 5:434-5.

45. Wang H, Qiu P, Liu J, Wang F, Zhao Q. The liver injury and gastrointestinal symptoms in patients with coronavirus disease 19: a systematic review and meta-analysis. Clin Res Hepatol Gastroenterol 2020; 44:653-61.

46. Cascella M, Rajnik M, Cuomo A, Dulebohn SC, Di Napoli R. Features, evaluation and treatment coronavirus (COVID-19). Treasure Island: StatPearls Publishing; 2020.

47. Servick K. For survivors of severe COVID-19, beating the virus is just the beginning. Science 2020; 8 abr. https://www.sciencemag. org/news/2020/04/survivors-severe-covid19-beating-virus-just-beginning.

48. Yang F, Shi S, Zhu J, Shi J, Dai K, Chen X. Analysis of 92 deceased patients with COVID-19. J Med Virol 2020; [Online ahead of print].

49. Fan Z, Chen L, Li J, Tian C, Zhang Y, Huang S, Liu Z, et al. Clinical features of COVID-19related liver functional abnormality. J Clin Gastroenterol Hepatol 2020; 18:1561-6.

50. Li J, Fan J-G. Characteristics and mechanism of liver injury in 2019 coronavirus disease. J Clin Transl Hepatol 2020; 8:13-7.

51. Giannis D, Ziogas IA, Gianni P. Coagulation disorders in coronavirus infected patients: COVID-19, SARS-CoV-1, MERS-CoV and lessons from the past. J Clin Virol 2020; 127:104362.

52. Lippi G, Plebani M, Henry BM. Thrombocytopenia is associated with severe coronavirus disease 2019 (COVID-19) infections: a metaanalysis. Clin Chim Acta 2020; 506:145-8.

53. Herridge MS, Tansey CM, Matté A, Tomlinson
G, Diaz-Granados N, Cooper A, et al. Functional disability 5 years after acute respiratory distress syndrome. N Engl J Med 2011; 364:1293-304.

54. Rawal G, Yadav S, Kumar R. Post-intensive care syndrome: an overview. J Transl Intern Med 2017; 5:90-2.

55. Robinson CC, Rosa RG, Kochhann R, Schneider D, Sganzerla D, Dietrich C, et al. Quality of life after intensive care unit: a multicenter cohort study protocol for assessment of longterm outcomes among intensive care survivors in Brazil. Rev Bras Ter Intensiva 2018; 30:40513.

56. Ramsay P, Huby G, Merriweather J, Salisbury L, Rattray J, Griffith D, et al. Patient and carer experience of hospital-based rehabilitation from intensive care to hospital discharge: mixed methods process evaluation of the RECOVER randomised clinical trial. BMJ Open 2016; 6:e012041.

57. Lonergan E, Luxenberg J, Areosa Sastre A. Benzodiazepines for delirium. Cochrane Database Syst Rev 2009; (1):CD006379.

58. Toubiana J, Poirault C, Corsia A, Bajolle F, Fougeaud J, Angoulvant F, et al. Kawasaki-like multisystem inflammatory syndrome in children during the covid-19 pandemic in Paris, France: prospective observational study. BMJ 2020; 369:m2094.

59. Whittaker E, Bamford A, Kenny J, Kaforou M, Jones CE, Shah P, et al. Clinical characteristics of 58 children with a pediatric inflammatory multisystem syndrome temporally associated with SARS-CoV-2. JAMA 2020; 324:259-69.

60. Toscano G, Palmerini F, Ravaglia S, Ruiz L, Invernizzi P, Cuzzoni MG, et al. Guillain-Barré syndrome associated with SARS-CoV-2. N Engl J Med 2020; 382:2574-6.

61. Needham EJ, Chou SH-Y, Coles AJ, Menon DK. Neurological implications of COVID-19 infections. Neurocrit Care 2020; 32:667-71.

62. Alberti P, Beretta S, Piatti M, Karantzoulis A, Piatti ML, Santoro P, et al. Guillain-Barré syndrome related to COVID-19 infection. Neurol Neuroimmunol Neuroinflamm 2020; 7:e741.

63. Camdessanche J-P, Morel J, Pozzetto B, Paul S, Tholance Y, Botelho-Nevers E. COVID-19 may induce Guillain-Barré syndrome. Rev Neurol (Paris) 2020; 176:516-8.

64. Marinho PM, Marcos AAA, Romano AC, Nascimento H, Belfort R. Retinal findings in patients with COVID-19. Lancet 2020; 395:1610.

65. Fan C, Li K, Ding Y, Lu WL, Wang J. ACE2 expression in kidney and testis may cause kidney and testis damage after $2019-\mathrm{NCoV}$ infection. medRxiv 2020; 13 fev. https://www.medrxiv. org/content/10.1101/2020.02.12.20022418v1.

66. Ozamiz-Etxebarria N, Dosil-Santamaria M, Picaza-Gorrochategui M, Idoiaga-Mondragon N. Niveles de estrés, ansiedad y depresión en la primera fase del brote del COVID-19 en una muestra recogida en el norte de España. Cad Saúde Pública 2020; 36:e00054020. 
67. Liu S, Yang L, Zhang C, Xiang Y-T, Liu Z, Hu $\mathrm{S}$, et al. Online mental health services in China during the COVID-19 outbreak. Lancet Psychiatry 2020; 7:e17-8.

68. The Lancet Psychiatry. Mental health and COVID-19: change the conversation. Lancet Psychiatry 2020; 7:463.

69. Camargo Jr. KR. Trying to make sense out of chaos: science, politics and the COVID-19 pandemic. Cad Saúde Pública 2020; 36:e00088120.

70. Girolamo G, Cerveri G, Clerici M, Monzani E, Spinogatti F, Starace F, et al. Mental health in the coronavirus disease 2019 emergency the Italian response. JAMA Psychiatry 2020; 77:974-6.

71. Druss BG. Addressing the COVID-19 pandemic in populations with serious mental illness. JAMA Psychiatry 2020; 77:891-2.

72. Tsamakis K, Rizos E, Manolis A, Chaidou S, Kympouropoulos S, Spartalis E, et al. COVID-19 pandemic and its impact on mental health of healthcare professionals. Exp Ther Med 2020; 19:3451-3.

73. Guterres A. We need to take action to address the mental health crisis. Time 2020; 21 may. https://time.com/5839553/un-action-mentalhealth-crisis/.

74. Shamasunder S, Holmes SM, Goronga T, Carrasco H, Katz E, Frankfurter R, et al. COVID-19 reveals weak health systems by design: why we must re-make global health in this historic moment. Glob Public Health 2020; $15: 1083-9$.

75. World Health Organization. Global health estimates 2016: deaths by cause, age, sex, by country and by region, 2000-2016. Geneva: World Health Organization; 2018.

76. Schramm JMA, Campos MR, Emmerick I, Sabino R, Sorio LF, Costa MFS, et al. Relatório de pesquisa: Projeto Carga do Diabetes e acesso ao tratamento e serviços de saúde no Estado do Rio Grande do Sul: um instrumento para gestão, organização e planejamento dos serviços de saúde. Rio de Janeiro: Fundação Oswaldo Cruz; 2018.

77. Schramm JM Andrade, Paes-Souza R, Mendes LVP. Políticas de austeridade e seus impactos na saúde. Rio de Janeiro: Centro de Estudos Estratégicos. Fundação Oswaldo Cruz; 2018. (Textos para Debate, 1).

78. Dweck E. Austeridade é a maior aliada do coronavírus no Brasil. Carta Maior 2020; 16 mar. https://www.cartamaior.com.br/?/ Editoria/Economia-Politica/Austeridadee-a-maior-aliada-do-coronavirus-no-Bra sil/7/46797.

79. Sands P, El Turabi A, Saynisch PA, Dzau VJ. Assessment of economic vulnerability to infectious disease crises. Lancet 2016; 388:2443-8.

80. Deccache D, Nascimento F, Ramos AP, Lemos I. Pandemia de coronavírus e a urgência da revogação do teto dos gastos. Brasil de Fato 2020; 16 mar. https://www.brasildefato.com. br/2020/03/16/artigo-pandemia-de-coro navirus-e-a-urgencia-da-revogacao-do-tetodos-gastos.
81. Castro CS, Holzgrefe Júnior JV, Reis RB, Andrade BB, Quintanilha LF. Pandemia da COVID-19: cenário do sistema de saúde brasileiro para o enfrentamento da crise. Research, Society and Development 2020; 9:516974383.

82. Hallal P, Hartwig F, Horta B, Victora GD, Silveira M, Struchiner C, et al. Remarkable variability in SARS-CoV-2 antibodies across Brazilian regions: Nationwide Serological Household Survey in 27 states. medRxiv 2020; 30 mai. https://www.medrxiv.org/content/10.11 01/2020.05.30.20117531v1.

83. Observatório COVID-19 Fiocruz. Análise da frequência, incidência, mortalidade e letalidade por COVID-19 em favelas cariocas. Boletim Socioepidemiológico da COVID-19 nas Favelas 2020; (1). https://portal.fiocruz.br/ sites/portal.fiocruz.br/files/documentos/bo letim_socioepidemiologicos_covid_nas_fave las_1.pdf.

84. Palmer K, Monaco A, Kivipelto M, Onder G, Maggi S, Michel J-P, et al. The potential longterm impact of the COVID-19 outbreak on patients with non-communicable diseases in Europe: consequences for healthy ageing. Aging Clin Exp Res 2020; 32:1189-94. .

85. Carvalho MS, Lima LD, Coeli CM. Ciência em tempos de pandemia. Cad Saúde Pública 2020; 36:e00055520.

86. Ministério da Saúde. Guia de vigilância epidemiológica. Emergência de saúde pública de importância nacional pela doença pelo coronavírus 2019 - vigilância integrada de síndromes respiratórias agudas. Brasília: Ministério da Saúde; 2020.

87. Secretaria de Atenção Primária à Saúde. Recomendações para adequação das ações dos agentes comunitários de saúde frente à atual situação epidemiológica referente à COVID-19. Brasília: Secretaria de Atenção Primária à Saúde; 2020.

88. Engstrom E, Melo E, Giovanella L, Mendes A, Grabois V. Recomendações para a Organização da Atenção Primária à Saúde no SUS no enfrentamento da Covid-19. Rio de Janeiro: Observatório COVID-19 Fiocruz; 2020. (Série Linha de Cuidado Covid-19 na Rede de Atenção à Saúde).

89. Vitória AM, Campos GWS. Só com APS forte o sistema pode ser capaz de achatar a curva de crescimento da pandemia e garantir suficiência de leitos UTI. https://frenteestamira.org/wpcontent/uploads/2020/04/A.Vit\%C3\%B3riaGast\%C3\%A3o-W.-S\%C3\%B3-APS-forte-as segura-achatar-a-curva-e-funcionamento-deleitos-de-UTI.-mar\%C3\%A7o2020.pdf (acessado em 28/Mai/2020). 


\section{Abstract}

COVID-19 is an acute infectious respiratory distress syndrome (ARDS) caused by the novel coronavirus SARS-CoV-2. The disease is highly communicable and produces mild to severe symptoms, generating a high demand for intensive care and thousands of deaths. In March 2020, COVID-19 was declared a pandemic and has already surpassed five million cases and 300,000 deaths in the world. The natural history of the disease has still not been fully established, hindering the elaboration of effective clinical protocols and preventive measures. Nevertheless, the disease requires a systemic approach, since there is evidence of acute and chronic complications, in addition to the catastrophic effects on the population's mental health. This highlights the need for a methodology that more effectively captures the effect of COVID-19, considering such aspects as severity, duration, and the potential to generate chronic complications that will increase the demands on Brazilian Unified National Health System (SUS). DALYs, or disability-adjusted life years, are thus an extremely useful indictor that adds mortality, an estimate of years of life lost (YLLs), and morbidity, an estimate of years of life lived with disability (YLDs). This article discusses the relevance and difficulties of studying the burden of COVID-19 and its complications in the Brazilian context, highlighting the natural history of the disease and estimating indicators such as YLDs, considering the high burden of disease in planning strategies to deal with the consequences of COVID-19 after the pandem$i c$. The article also discusses the future challenges to deal with the disease in the SUS and the effects on the calculation of DALYs.

Coronavirus Infections; Potential Years of Life Lost; Indicator of Morbidity and Mortality; Primary Health Care

\section{Resumen}

La COVID-19 es un síndrome respiratorio agudo grave (SRAG) infeccioso, causado por coronavirus. La enfermedad posee una alta transmisibilidad y ocasiona de sintomas leves a graves, generando una elevada demanda de cuidados intensivos y millares de fallecimientos. En marzo de 2020, la COVID-19 se caracterizó como pandemia y ya suma más de 5 millones de casos y 300 mil fallecimientos por el mundo. La historia natural de la enfermedad todavía no ha sido bien establecida, dificultando la elaboración de protocolos clínicos eficaces y medidas de prevención. A pesar de eso, se puede afirmar que es una enfermedad de abordaje sistémico, ya que existen evidencias sobre complicaciones agudas y crónicas, además de efectos catastróficos en la salud mental de la población. Se destaca entonces la necesidad de una metodología que capte de forma más efectiva los efectos de la COVID-19, considerando aspectos como su gravedad, duración, potencial de generar complicaciones crónicas que aumentarán las demandas en el Sistema Único de Salud (SUS). En este sentido, es de extrema utilidad el indicador $D A L Y$ o años de vida perdidos por muerte prematura ajustados por incapacidad, que agrega la (1) mortalidad - estimación de los años de vida perdidos (YLL) y (2) morbilidad - estimación de los años vividos con incapacidad (YLD). Este artículo discute la relevancia y las dificultades de estudiar la carga de la COVID-19y sus complicaciones, en el contexto brasileño, resaltando la importancia de caracterizar la historia natural de la enfermedad y estimar indicadores como el YLD, que consideren la alta carga de morbilidad en la planificación de estrategias para lidiar con las consecuencias de la COVID-19 pospandemia. Se discuten también los desafíos futuros para el combate de la enfermedad en el SUS y reflexiones sobre el cálculo del DALY.

Infecciones por Coronavírus; Años Potenciales de Vida Perdidos; Indicadores de Morbimortalidad; Atención Primaria de Salud
Recebido em 02/Jun/2020

Versão final reapresentada em 21/Jul/2020

Aprovado em 24/Jul/2020 